\title{
Universiteit
}

Leiden

The Netherlands

\section{Manipulation of photon statistics of highly degenerate incoherent radiation}

Kindermann, M.; Nazarov, Y.V.; Beenakker, C.W.J.

\section{Citation}

Kindermann, M., Nazarov, Y. V., \& Beenakker, C. W. J. (2002). Manipulation of photon statistics of highly degenerate incoherent radiation. Physical Review Letters, 88(6), 063601. doi:10.1103/PhysRevLett.88.063601

Version: $\quad$ Not Applicable (or Unknown)

License: $\quad$ Leiden University Non-exclusive license

Downloaded from: https://hdl.handle.net/1887/66534

Note: To cite this publication please use the final published version (if applicable). 


\title{
Manipulation of Photon Statistics of Highly Degenerate Incoherent Radiation
}

\author{
M. Kindermann, ${ }^{1}$ Yu. V. Nazarov, ${ }^{2}$ and C. W. J. Beenakker ${ }^{1}$ \\ ${ }^{1}$ Instituut-Lorentz, Universiteit Leiden, P.O. Box 9506, 2300 RA Leiden, The Netherlands \\ ${ }^{2}$ Department of Applied Physics and Delft Institute of Microelectronics and Submicrontechnology, \\ Delft University of Technology, Lorentzweg 1, 2628 CJ Delft, The Netherlands
}

(Received 5 July 2001; published 25 January 2002)

\begin{abstract}
Highly degenerate incoherent radiation has a Gaussian density matrix and a large occupation number of modes $f$. If it is passed through a weakly transmitting barrier, its counting statistics is close to Poissonian. We show that a second identical barrier, in series with the first, drastically modifies the statistics. The variance of the photocount is increased above the mean by a factor $f$ times a numerical coefficient. The photocount distribution reaches a limiting form with a Gaussian body and highly asymmetric tails. These are general consequences of the combination of weak transmission and multiple scattering.
\end{abstract}

DOI: 10.1103/PhysRevLett.88.063601

Chaotic radiation is the name given in quantum optics to a gas of photons that has a Gaussian density matrix [1]. (To avoid misunderstanding, we note that chaotic radiation is not in any way related to chaos in classical mechanics.) The radiation emitted by a black body is a familiar example. The statistics of black-body radiation, as measured by a photodetector, is very close to the Poisson statistics of a gas of classical independent particles. Deviations due to photon bunching exist, but these are small corrections. To see effects of Bose statistics one needs a degenerate [2] photon gas, with an occupation number $f$ of the modes that is $\gtrsim 1$. Black-body radiation at optical frequencies is nondegenerate to a large degree $\left(f \simeq e^{-\hbar \omega / k T} \ll 1\right)$, even at temperatures reached on the surface of the Sun.

The degeneracy is no longer restricted by frequency and temperature if the photon gas is brought out of thermal equilibrium. The coherent radiation from a laser would be an extreme example of high degeneracy, but the counting statistics is still Poissonian because of the special properties of a coherent state [1]. One way to create nonequilibrium chaotic radiation is spectral filtering within the quantum-limited linewidth of a laser [3]. This will typically be single-mode radiation. For multimode radiation one can pass black-body radiation through a linear amplifier. The amplification might be due to stimulated emission by an inverted atomic population or to stimulated Raman scattering [4]. Alternatively, one can use the spontaneous emission from an amplifying medium that is well below the laser threshold [5], or parametric down-conversion in a nonlinear crystal [1].

The purpose of this paper is to show that the statistics of degenerate chaotic radiation can be manipulated by introducing scatterers, to an extent that would be impossible for both nondegenerate chaotic radiation and degenerate coherent radiation. We will illustrate the difference by examining in some detail a simple geometry consisting of one or two weakly transmitting barriers (in analogy with tunnel barriers for electrons) [6] embedded in a waveguide (see Fig. 1). For the single barrier the photocount distribution is close to Poissonian. The mean photocount $\bar{n}$ is changed
PACS numbers: 42.50.Ar, 42.25.Bs, 42.50.Lc

by only a factor of 2 upon insertion of the second barrier. But the fluctuations around the mean are greatly enhanced, as a result of multiple scattering in a region of large occupation number. We find that the distribution $P(n)$ for the double-barrier geometry is not only much broader than a Poisson distribution, it also has a markedly different shape.

We consider a source of chaotic radiation that is not in thermal equilibrium. Chaotic radiation is characterized by a Gaussian density matrix $\rho$ in the coherent state representation [1]. For a single mode it takes the form

$$
\rho=\int d \alpha d \alpha^{*}(\pi \mu)^{-1} \exp \left(-\alpha^{*} \mu^{-1} \alpha\right)|\alpha\rangle\langle\alpha|,
$$

where $\mu$ is a positive real number and $|\alpha\rangle$ is a coherent state (eigenstate of the photon annihilation operator

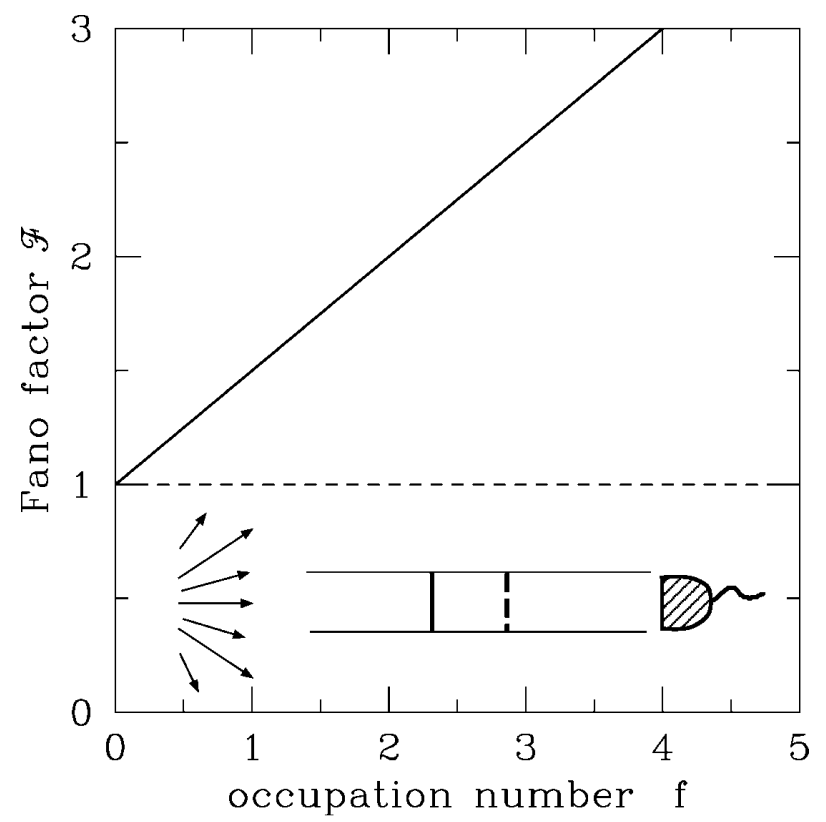

FIG. 1. Dependence of the Fano factor on the occupation number of the modes, for transmission through one (dashed line) or two barriers (solid line). The inset shows schematically the photodetector (shaded) and the waveguide containing one or two barriers. 
a) with complex eigenvalue $\alpha$. If one takes into account more modes, $\alpha$ becomes a vector $\boldsymbol{\alpha}$ and $\mu$ a matrix $\boldsymbol{\mu}$ in the space of modes. (The factor $\pi \mu$ then becomes the determinant $\|\pi \boldsymbol{\mu}\|$.) We take a waveguide geometry and assume that the radiation is restricted to a narrow frequency interval $\delta \omega$ around $\omega_{0}$. In this case the indices $n, m$ of $\alpha_{n}, \mu_{m n}$ label the $N$ propagating waveguide modes at frequency $\omega_{0}$.

In thermal equilibrium at temperature $T$, the covariance matrix $\boldsymbol{\mu}=f \mathbb{1}$ equals the unit matrix $\mathbb{1}$ times the scalar factor $f=\left(e^{\hbar \omega / k T}-1\right)^{-1}$, being the Bose-Einstein distribution function. Multimode chaotic radiation out of thermal equilibrium has in general a nonscalar $\boldsymbol{\mu}$. We assume that $\boldsymbol{\mu}$ is a property of the amplifying medium, independent of the scattering properties of the waveguide to which it is coupled. Feedback from the waveguide into the amplifier is therefore neglected.

The radiation is fully absorbed at the other end of the waveguide by a photodetector. We seek the probability distribution $P(n)$ to count $n$ photons in a time $t$. It is convenient to work with the cumulant generating function $F(\xi)=\ln \left[\sum_{n} e^{\xi n} P(n)\right]$. For long counting times $t \delta \omega \gg 1$ it is given by the Glauber formula [1,7]

$$
F(\xi)=\frac{t \delta \omega}{2 \pi} \ln \operatorname{Tr}\left(\rho: \exp \left[\left(e^{\xi}-1\right) \mathbf{a}_{\text {out }}^{\dagger} \mathbf{a}_{\text {out }}\right]:\right) .
$$

Here $\boldsymbol{a}_{\text {out }}$ is the vector of annihilation operators for the modes going out of the waveguide and into the photodetector. The colons : : indicate normal ordering (creation operators to the left of annihilation operators). The transmission matrix $\boldsymbol{t}$ relates $\boldsymbol{a}_{\text {out }}=\boldsymbol{t} \boldsymbol{a}$ to the vector $\boldsymbol{a}$ of annihilation operators entering the waveguide. Substituting Eq. (1) for $\rho$, we find

$$
\begin{aligned}
F(\xi)= & \frac{t \delta \omega}{2 \pi} \ln \int d \boldsymbol{\alpha} d \boldsymbol{\alpha}^{*}\|\pi \boldsymbol{\mu}\|^{-1} \exp \left(-\boldsymbol{\alpha}^{*} \boldsymbol{\mu}^{-1} \boldsymbol{\alpha}\right) \\
& \times \exp \left[\left(e^{\xi}-1\right) \boldsymbol{\alpha}^{*} t^{\dagger} \boldsymbol{t} \boldsymbol{\alpha}\right] \\
= & -\frac{t \delta \omega}{2 \pi} \ln \left\|\mathbb{1}-\left(e^{\xi}-1\right) \boldsymbol{\mu} t^{\dagger} \boldsymbol{t}\right\|
\end{aligned}
$$

In thermal equilibrium, when $\boldsymbol{\mu}=f \mathbb{1}$, the determinant can be evaluated in terms of the eigenvalues $T_{n}$ of the matrix product $\boldsymbol{t}^{\dagger} \boldsymbol{t}$. The resulting expression $[5,8]$

$$
F(\xi)=-\frac{t \delta \omega}{2 \pi} \sum_{n=1}^{N} \ln \left[1-\left(e^{\xi}-1\right) f T_{n}\right]
$$

has a similar form as the generating function of the electronic charge counting distribution at zero temperature [9],

$$
F_{\text {electron }}(\xi)=\frac{t e V}{2 \pi \hbar} \sum_{n=1}^{N} \ln \left[1+\left(e^{\xi}-1\right) T_{n}\right],
$$

where $V$ is the applied voltage

If the eigenvalues of $\boldsymbol{t} \boldsymbol{\mu} \boldsymbol{t}^{\dagger}$ are $\ll 1$, we may expand the logarithm in Eq. (3) to obtain $F(\xi)=\bar{n}\left(e^{\xi}-1\right)$, with mean photocount $\bar{n}=(t \delta \omega / 2 \pi) \operatorname{Tr} \boldsymbol{\mu} t^{\dagger} \boldsymbol{t}$. The corresponding photocount distribution is Poissonian,

$$
P_{\text {Poisson }}(n)=\frac{1}{n !} \bar{n}^{n} e^{-\bar{n}} .
$$

In thermal equilibrium the deviations from a Poisson distribution will be very small, because the Bose-Einstein function is $\ll 1$ at optical frequencies for any realistic temperature. There is no such restriction on the covariance matrix $\boldsymbol{\mu}$ out of equilibrium. This leads to striking deviations from Poisson statistics.

As a measure for deviations from a Poisson distribution we consider the deviations from unity of the Fano factor. From Eq. (4) we derive

$$
\mathcal{F}=\frac{\operatorname{Var} n}{\bar{n}}=1+\frac{\operatorname{Tr}\left(\boldsymbol{\mu} \boldsymbol{t}^{\dagger} \boldsymbol{t}\right)^{2}}{\operatorname{Tr} \boldsymbol{\mu} \boldsymbol{t}^{\dagger} \boldsymbol{t}} .
$$

A Fano factor $\mathcal{F}>1$ indicates photon bunching. For example, for black-body radiation $\mathcal{F}=1+f$. One might surmise that photon bunching is negligible if the waveguide is weakly transmitting, so that $N^{-1} \operatorname{Tr} t^{\dagger} t \ll 1$. That is correct if the weak transmission is due to a single barrier. Then each transmission eigenvalue $T_{n} \ll 1$, hence $\mathcal{F} \approx 1$. However, if a second identical barrier is placed in series with the first one a remarkable increase in the Fano factor occurs.

Let us first demonstrate this effect for a scalar $\boldsymbol{\mu}=$ $f \mathbb{1}$, when it has a well-known electronic analog $[10,11]$. We assume that $N \gg 1$ so that we may replace traces in Eq. (7) by integrations over the transmission eigenvalue $T$ with density $\rho(T)$,

$$
\mathcal{F}=1+f \frac{\int_{0}^{1} d T \rho(T) T^{2}}{\int_{0}^{1} d T \rho(T) T} .
$$

For a single barrier $\rho(T)$ is sharply peaked at a transmittance $\Gamma \ll 1$. Hence, $\mathcal{F} \approx 1$ for a single barrier. For two identical barriers in series the density is bimodal [12],

$$
\rho(T)=\frac{N \Gamma}{2 \pi} T^{-3 / 2}(1-T)^{-1 / 2},
$$

with a peak near $T=0$ and at $T=1$. From this distribution we find that

$$
\mathcal{F}=1+\frac{1}{2} f .
$$

While the second barrier reduces the mean photocount by only a factor of 2 , independently of the occupation number $f$ of the modes of the incident radiation, it can greatly increase the Fano factor for large $f$ (see Fig. 1). From the electronic analog (5) we would find $\mathcal{F}=1$ for a single barrier and $\mathcal{F}=1-\frac{1}{2}=\frac{1}{2}$ for a double barrier [10]. We conclude that for electrons the effect of the second barrier on the mean current and the Fano factor are comparable (both being a factor of 2), while for photons the effect on the Fano factor can be orders of magnitude greater than on the mean current for $f \gg 1$.

The two terms 1 and $\frac{1}{2} f$ in Eq. (10) account, respectively, for the particle and the wave nature of the radiation. For a classical wave the mean of the squared intensity fluctuations is proportional to the mean intensity squared, 
hence a classical wave has a Fano factor that varies linearly with $f$. In the double barrier geometry there is a high intensity of the radiation in a region with strong multiple scattering, and this enhances the wave contribution to $\mathcal{F}$ relative to the particle contribution. This explains in simple terms why $\mathcal{F} \propto f$ for $f \gg 1$, but to find the numerical coefficient $\frac{1}{2}$ and the crossover to particlelike behavior relevant in the single-barrier geometry requires an explicit calculation.

Changing the nature of the multiple scattering will change the numerical coefficient. For example, multiple scattering by disorder would give $\mathcal{F}=1+\frac{2}{3} f$, in analogy with the electronic result $[13,14] \mathcal{F}=1-\frac{2}{3}=\frac{1}{3}$. What the double-barrier and the disordered cases have in common is a $\rho(T)$ that is very broad. (Typically it is bimodal, with peaks at $T=0,1$.) The shape of the distribution depends on the type of multiple scattering, and that in turn affects the numerical coefficients, but the coefficient remains of order unity. [The single barrier, in contrast, has a unimodal $\rho(T)$, all transmission probabilities are concentrated around $T=\Gamma$.] The bimodal $\rho(T)$ can be understood as being a precursor of wave localization due to multiple scattering [15]. The bimodal $\rho(T)$ does not depend on the separation $L$ of the barriers, as long as it is large compared to the wavelength $\lambda$ and short compared to the absorption length $\xi$. For $L \lesssim \lambda$ we are back to the single-barrier case and for $L \gg \xi$ the Fano factor tends to zero.

We now generalize Eq. (10) to a nonscalar $\boldsymbol{\mu}$. An extreme case is a covariance matrix of rank one having all eigenvalues $\mu_{n}$ equal to zero except a single one. This would happen if the waveguide is far removed from the source, so that its cross-sectional area $A$ is smaller than the coherence area $A_{c}$ [16]. Since $\operatorname{Tr}\left(\boldsymbol{\mu} \boldsymbol{t}^{\dagger} \boldsymbol{t}\right)^{2}=\left(\operatorname{Tr} \boldsymbol{\mu} \boldsymbol{t}^{\dagger} \boldsymbol{t}\right)^{2}$ if $\boldsymbol{\mu}$ is of rank one, the Fano factor reduces to $\mathcal{F}=$ $1+\operatorname{Tr} \boldsymbol{\mu} \boldsymbol{t}^{\dagger} \boldsymbol{t}$. The trace of $\boldsymbol{\mu} \boldsymbol{t}^{\dagger} \boldsymbol{t}$ is $\ll 1$ for both singleand double-barrier geometry, hence a second barrier has no large effect on the noise if $A \lesssim A_{c}$.

More generally, for a nonscalar $\boldsymbol{\mu}$ the Fano factor (7) depends not just on the eigenvalues $T_{n}$ of $\boldsymbol{t}^{\dagger} \boldsymbol{t}$, but also on the eigenvectors. We write $\boldsymbol{t}^{\dagger} \boldsymbol{t}=\boldsymbol{U}^{\dagger} \boldsymbol{\tau} \boldsymbol{U}$, with $\boldsymbol{U}$ the unitary matrix of eigenvectors. We assume strong intermode scattering by disorder inside the waveguide. The resulting $\boldsymbol{U}$ will then be uniformly distributed in the unitary group, independent of $\boldsymbol{\tau}$ [15]. For $N \gg 1$ we can replace the traces in numerator and denominator in Eq. (7) by integrations over $\boldsymbol{U}$, with the result

$$
\mathcal{F}=1+\langle\boldsymbol{\mu}\rangle\langle\boldsymbol{\tau}\rangle+\langle\boldsymbol{\mu}\rangle \frac{\left\langle\left\langle\boldsymbol{\tau}^{2}\right\rangle\right\rangle}{\langle\boldsymbol{\tau}\rangle}+\langle\boldsymbol{\tau}\rangle \frac{\left\langle\left\langle\boldsymbol{\mu}^{2}\right\rangle\right\rangle}{\langle\boldsymbol{\mu}\rangle} .
$$

Here $\left\langle\boldsymbol{\mu}^{p}\right\rangle=N^{-1} \operatorname{Tr} \boldsymbol{\mu}^{p},\left\langle\boldsymbol{\tau}^{p}\right\rangle=N^{-1} \operatorname{Tr} \boldsymbol{\tau}^{p}$ denote the spectral moments and $\left\langle\left\langle\boldsymbol{\mu}^{p}\right\rangle\right\rangle,\left\langle\left\langle\boldsymbol{\tau}^{p}\right\rangle\right\rangle$ the corresponding cumulants. (For example, $\left\langle\left\langle\boldsymbol{\tau}^{2}\right\rangle\right\rangle=\left\langle\boldsymbol{\tau}^{2}\right\rangle-\langle\boldsymbol{\tau}\rangle^{2}$.)

Instead of Eq. (10) we now have for the double-barrier geometry a Fano factor

$$
\mathcal{F}=1+\frac{1}{2}\langle\boldsymbol{\mu}\rangle(1+\kappa), \quad \kappa=\Gamma \frac{\left\langle\left\langle\boldsymbol{\mu}^{2}\right\rangle\right\rangle}{\langle\boldsymbol{\mu}\rangle^{2}} .
$$

We may estimate the magnitude of the correction $\kappa$ by noting that, typically, only $N_{c} \simeq A / A_{c}$ eigenvalues of $\boldsymbol{\mu}$ will be significantly different from 0 . If we ignore the spread among these $N_{c}$ eigenvalues, we have $\left\langle\boldsymbol{\mu}^{2}\right\rangle \approx$ $\left(N / N_{c}\right)\langle\boldsymbol{\mu}\rangle^{2}$; hence $\kappa \approx \Gamma\left(N / N_{c}-1\right)$. This correction will be negligibly small for $\Gamma \ll 1$, unless $\Gamma N \gtrsim N_{c}$.

In the final part of this paper we consider the full photocount probability distribution $P(n)=$ $(2 \pi)^{-1} \int_{0}^{2 \pi} d \xi \exp [F(i \xi)-i n \xi]$. For large detection time this integral can be done in saddle point approximation. The result has the form $P(n)=\exp [\bar{n} g(n / \bar{n})]$. For small relative deviations of $n$ from $\bar{n}$ the function $g(n / \bar{n})$ can be expanded to second order in $n / \bar{n}$. Thus the body of the distribution tends to a Gaussian for $t \rightarrow \infty$, in accordance with the central limit theorem. The same holds for the Poisson distribution (6). However, the tails of $P(n)$ for degenerate radiation remain non-Gaussian and different from the tails of $P_{\text {Poisson }}(n)$.

Let us first investigate this for a scalar $\boldsymbol{\mu}=f \mathbb{1}$. Replacing the sum over $n$ in Eq. (4) by the integral $\int_{0}^{1} d T \rho(T)$, which is allowed in the large- $N$ limit, we find, using Eq. (9), the generating function

$$
F(\xi)=\frac{t \delta \omega}{2 \pi} N \Gamma\left[1-\sqrt{1-\left(e^{\xi}-1\right) f}\right] .
$$

The corresponding $P(n)$ is the $K$ distribution that has appeared before in a variety of contexts $[8,17]$. The $K$ distribution is usually considered only for $f \ll 1$, as is appropriate for thermal equilibrium. In the regime $1 \ll f \ll \bar{n}$ of interest here it has the form

$$
P(n)=C n^{-3 / 2} \exp \left(-\frac{n}{f}-\frac{\bar{n}^{2}}{n f}\right),
$$

with a normalization constant $C=\bar{n}(\pi f)^{-1 / 2} \exp (2 \bar{n} / f)$. The essential singularity at $n=0$ is cut off below $\bar{n} / \sqrt{f}$, where the distribution saturates at $P(0)=\exp (-2 \bar{n} / \sqrt{f})$.

In Fig. 2 we compare the distribution (14) with a Gaussian and with a Poisson distribution, which has the asymptotic form $P_{\text {Poisson }}=(2 \pi n)^{-1 / 2} \exp [n-\bar{n}-n \ln (n / \bar{n})]$. The logarithmic plot emphasizes the tails, which are markedly different.

For a nonscalar $\boldsymbol{\mu}$ we find that the functional form of the large- $n$ tail depends only on the largest eigenvalue $\lambda_{\max } \gg 1$ of the Hermitian positive definite matrix $\boldsymbol{t} \boldsymbol{\mu} \boldsymbol{t}^{\dagger}$,

$$
\lim _{n \rightarrow \infty} P(n) \propto e^{-n / \lambda_{\max }} .
$$

The number $\lambda_{\max }$ plays the role for a nonscalar $\boldsymbol{\mu}$ of the filling factor $f$ in the result (14) for a scalar $\boldsymbol{\mu}$. While the large- $n$ tail is exponential under very general conditions, the tail for $n \ll \bar{n}$ has no universal form.

In conclusion, we have calculated the effect of multiple scattering on the photodetection statistics of radiation that is both chaotic (like thermal radiation from a black 


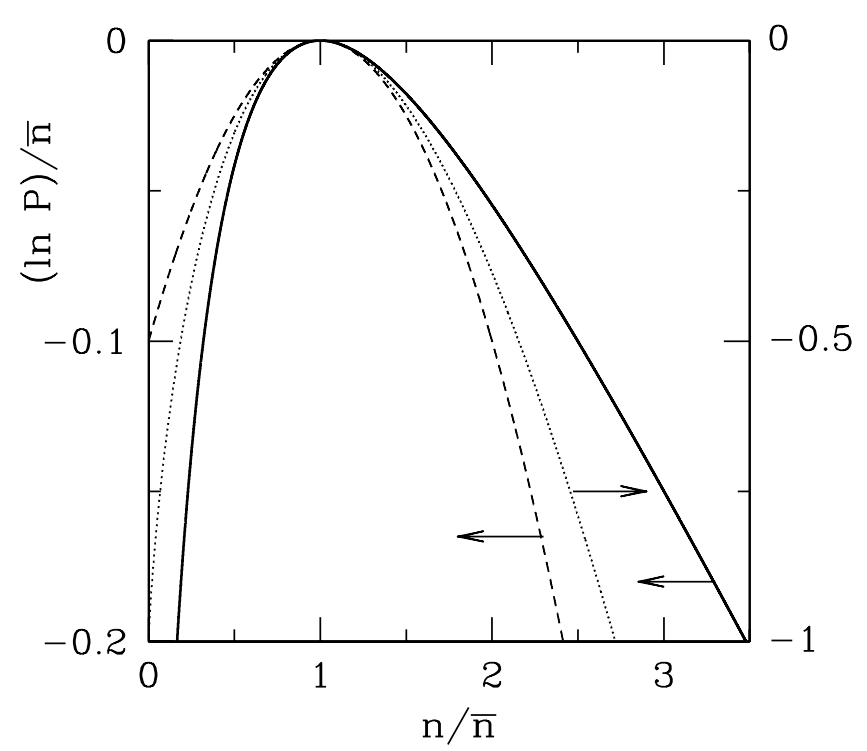

FIG. 2. Logarithmic plot of the photocount distribution for $f=8$ and $\bar{n} \rightarrow \infty$. The solid curve follows from Eq. (13) (describing the double-barrier geometry) and is very close to the large- $f$ limit (14). The dashed curve is a Gaussian with variance $\left(1+\frac{1}{2} f\right) \bar{n}$, and the dotted curve is the Poisson distribution (6). (Notice the different vertical scale for the dotted curve, chosen such that the Gaussian body of the Poisson distribution becomes evident.)

body) and highly degenerate (like coherent radiation from a laser). Even for weak transmission there appear large deviations of the photocount distribution from Poisson statistics that are absent in the radiation from a black body or a laser. They take the form of an enhancement of Varn above $\bar{n}$ by a factor $\propto f$ and a slowing down of the large- $n$ decay rate of $P(n)$ by a factor $1 / f$. Explicit results have been given for a double barrier geometry, but these findings are generic and would apply also, for example, to multiple scattering by disorder. Because of this generality we believe that experimental observation of our predictions would be both significant and feasible.

This work was supported by the Dutch Science Foundation NWO/FOM.
[1] L. Mandel and E. Wolf, Optical Coherence and Quantum Optics (Cambridge University Press, Cambridge, 1995).

[2] We use the word "degenerate" here by analogy with the degenerate electron gas.

[3] R. Centeno Neelen, D. M. Boersma, M. P. van Exter, G. Nienhuis, and J. P. Woerdman, Phys. Rev. Lett. 69, 593 (1992).

[4] C. H. Henry and R. F. Kazarinov, Rev. Mod. Phys. 68, 801 (1996).

[5] C. W. J. Beenakker, Phys. Rev. Lett. 81, 1829 (1998).

[6] As a barrier one could use a "spatial filter" consisting of a metal perforated by a large number of subwavelength holes. Absorption by the metal should be minimized because it suppresses the multiple scattering that is at the origin of the effect predicted here. Another realization would be a layered medium with a refractive index that is randomly distributed along the direction of light propagation. There the light intensity would decay exponentially due to wave localization.

[7] R. J. Glauber, Phys. Rev. Lett. 10, 84 (1963).

[8] C. W. J. Beenakker, in Diffuse Waves in Complex Media, edited by J.-P. Fouque, NATO ASI, Ser. C, Vol. 531 (Kluwer, Dordrecht, 1999).

[9] L. S. Levitov and G. B. Lesovik, JETP Lett. 58, 230 (1993).

[10] L. Y. Chen and C. S. Ting, Phys. Rev. B 43, 4534 (1991).

[11] Ya. M. Blanter and M. Büttiker, Phys. Rep. 336, 1 (2000).

[12] J. A. Melsen and C. W. J. Beenakker, Physica (Amsterdam) 203B, 219 (1994). The distribution (9) requires $N \gg$ $\Gamma^{-1} \gg 1$. For a spatial filter with one hole per wavelength squared one can identify $\Gamma$ with the transmittance of a single hole and $N$ with the total number of holes.

[13] C. W. J. Beenakker and M. Büttiker, Phys. Rev. B 46, 1889 (1992).

[14] K. E. Nagaev, Phys. Lett. A 169, 103 (1992).

[15] C. W. J. Beenakker, Rev. Mod. Phys. 69, 731 (1997).

[16] The coherence area $A_{c} \approx R^{2} / N_{\text {source }}$ of multimode radiation increases quadratically with separation $R$ from the source ( $N_{\text {source }}$ being the number of modes).

[17] M. Bertolotti, B. Crosignani, and P. Di Porto, J. Phys. A 3, L37 (1970); E. Jakeman and P. N. Pusey, Phys. Rev. Lett. 40, 546 (1978). In these two papers the noise is due to time-dependent fluctuations in the scattering medium. In contrast, we consider time-dependent fluctuations of the photon flux in the presence of static scatterers. 